\title{
De Novo Donor-SPECIFIC ANTIBOdies AfTER Allograft NePHRECTOMY
}

Gaetano Lucisano ${ }^{1}$, Paul Brookes ${ }^{2}$, Eva Santos-Nunez², Nicola Firmin², Nicola Gunby², Sevda Hassan¹, Alexander Gueret-Guardle ${ }^{1}$, Michelle Willicombe ${ }^{1}$, David Taube ${ }^{1}$

${ }^{1}$ Renal and Transplant Centre, Imperial College Healthcare NHS Trust, London

${ }^{2}$ Histocompatibility and Immunogenetics, Imperial College Healthcare NHS Trust, London

\section{INTRODUCTION}

Allograft nephrectomy (NX) is a well recognised cause of HLA sensitisation after graft failure [1] and it has shown to be an independent predictor for the development of DSA after ceasing immunosuppressive therapy in patients with failed grafts [2]. The presence of pre-formed DSAs is associated with poor allograft outcomes [3] and this should be taken into account for patients awaiting re-transplantation. As yet, there are few studies describing the tempo, class and predictors of DSA development in previously unsensitised patients undergoing NX after the failure of their first graft.

\section{METHODS}

263/1,714 (15.3\%) patients transplanted at our Centre between August 2004 and October 2015 lost their grafts and 106 underwent NX. Only patients returning to dialysis after the failure of their first graft and without detectable DSA at the time of NX or transplant failure were included. Patients were divided into two groups according to whether they underwent NX after transplant failure (NX+) or not (NX-). Sera were taken for class I HLA (A/B/Cw) and class II (DR/DP/DQ) at the time of NX (at the time of transplant failure for the NX-group) and after 3, 6, 12 and 24 months using the single antigen Luminex assay. A mean fluorescence intensity value of $>1,000$ was considered positive.

Statistical analysis was performed using IBM SPSS Statistics ver. 20 and GraphPad Prism ver. 6.0

\section{RESULTS}

63 and 49 patients were assigned to group NX+ and NX- respectively (Table 1). Tacrolimus (Tac) was discontinued at the time of NX in 51/63 and by 3 months after NX in 12/63 patients, but continued in 47/49 NX-patients. As expected, we observed a higher rate of DSA positive patients in the NX+ group compared to the NX- group ( $\mathrm{p}<0.001$ for all the study time points, (Figure 1). DSA formation (class I, II and I+II) was significantly greater $(\mathrm{p}<0.001)$ in $\mathrm{NX}+$ group and DSA formation was delayed in the NX-group.(Figure 2). Tac withdrawal in both groups was associated with an increased incidence of DSA production ( $\mathrm{p}<0.001$ at all time points, (Figure 3). After correction for previous pregnancies, blood transfusions, mismatch and transplant vintage, multivariate analysis revealed the NX as a risk factor for the production of DSAs after 6,12 and 24 month and the pre-transplant dialysis duration and history of rejection as risk factors for the development of DSA after 24 months.

\begin{tabular}{|c|c|c|c|}
\hline Table 1 & $N X+(N=63)$ & $N X-(N=49)$ & p \\
\hline Female (\%) & 53.7 & 46.3 & 0.697 \\
\hline Previous pregnancies (\%) & 20.6 & 18.4 & 0.814 \\
\hline Previous transfusions (\%) & 93.7 & 83.7 & 0.125 \\
\hline$>5$ transfusions $(\%)$ & 50.0 & 20.4 & 0.003 \\
\hline Pre-emptive (\%) & 22.2 & 20.4 & 0.816 \\
\hline Pre-Tx RRT duration, months & $18.1(0.1-77.6)$ & $22.8(2.1-72.7)$ & 0.635 \\
\hline Live-donor (\%) & 44.4 & 32.7 & 0.244 \\
\hline Alemtuzumab (\%) & 65.1 & 79.6 & 0.139 \\
\hline History of rejection (\%) & 31.7 & 24.5 & 0.527 \\
\hline History of AMR (\%) & 28.6 & 20.4 & 0.383 \\
\hline Tx vintage, days & 209 (4-958) & $1,805(863-2,512)$ & $<0.001$ \\
\hline $\begin{array}{c}\text { NHSBT MM: } \\
\text { "000» } \\
\text { «Favourable» } \\
\text { "Unfavourable» }\end{array}$ & $\begin{array}{c}6.3 \\
71.4 \\
22.2\end{array}$ & $\begin{array}{l}14.3 \\
49.0 \\
36.7\end{array}$ & 0.048 \\
\hline Total MM, median & $3(2-4)$ & $4(2-5)$ & 0.265 \\
\hline
\end{tabular}

\section{CONCLUSIONS}

In our selected cohort of unsensitised patients with a first failed kidney, graft NX was followed by significant and continuing DSA production compared with NX- patients. However NX-patients did develop DSA 6 to 12 months after graft failure, albeit less frequently.

Our results suggest that NX and immunosuppression withdrawal for non essential reasons should be avoided particularly in patients suitable for retransplantation.

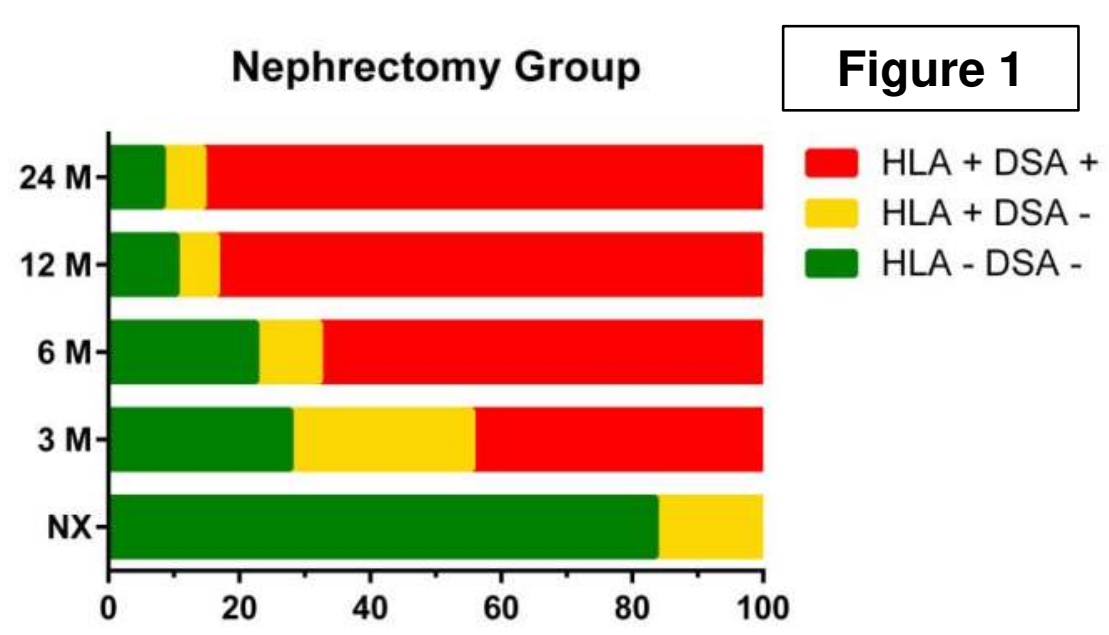

No Nephrectomy Group
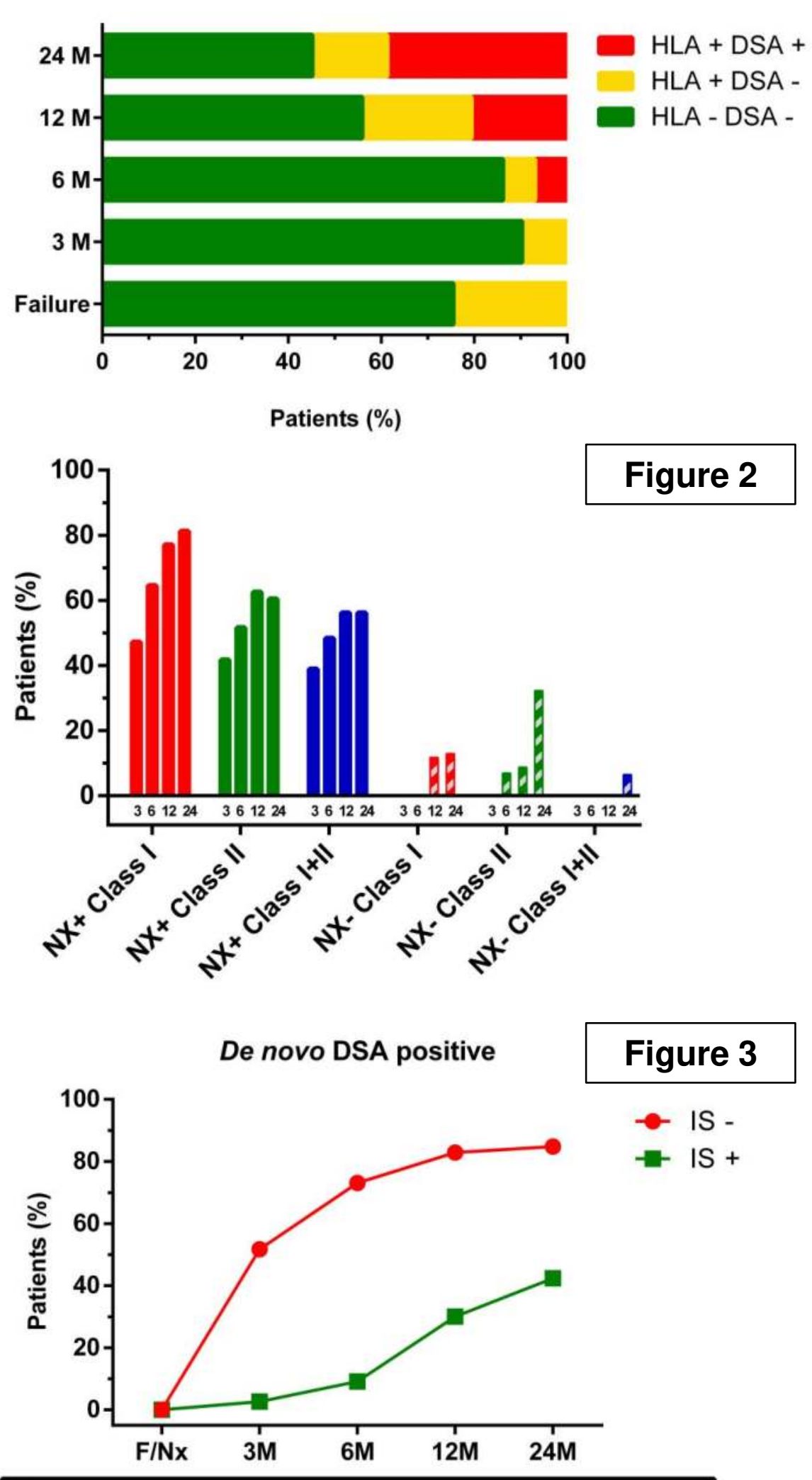

-: immunosuppression discontinued at the time of $\mathrm{Nx}$ or within 12 months after failure in NX-patients

S + : immunosuppression continued up to 3 months after $\mathrm{Nx}$ or not discontinued in NX- patients

\section{REFERENCES}

[1] Khakhar AK et al, Transplant Proc 2003; 35: 862

[2] Del Bello A et al, CJASN 2012;7:1310-1319

[3] Lefaucheur et al, American Journal of Transplantation 2008; 8-324

Imperial College

KIDNEY \& TRANSPLANT CENTRE

NIHR Imperial Biomedical Research Centre Translating research into patient benefits 